Background Recently bronchopulmonary dysplasia (BPD) occurs not infrequently without an exposure to hyperoxia and/or mechanical ventilation in very preterm infants. To simulate the recent pattern of BPD, novel animal model of BPD induced by inflammation alone is needed. We were to establish a rat model of BPD induced by postnatal systemic lipopolysaccharide (LPS) administration alone.

Methods Two days before delivery, $1 \mu \mathrm{g}$ of LPS or vehicle (V) was injected into each amniotic sac, and after birth $0.25 \mathrm{mg} / \mathrm{kg}$ of LPS or V was injected into peritoneum of pups at P1, P3, and $\mathrm{P} 5$. At $\mathrm{P} 7$ and $\mathrm{P} 14$, bronchoalveolar lavage (BAL) and lung harvest were performed. BAL fluid (BALF) and peripheral blood $(\mathrm{PB})$ were examined for blood cell counts and lung tissue was examined for morphometry, vascular density, neutrophil infiltration, and expressions of pro-inflammatory cytokines and angiogenic growth factors.

Results Postnatal systemic LPS significantly increased the neutrophil counts in PB, BALF and within the alveoli and expressions of pro-inflammatory cytokines and angiogenic growth factors at P7. When postnatal systemic LPS was preceded by intra-amniotic LPS administration, these findings were not observed. Postnatal systemic LPS led to significant disruption of alveolar and pulmonary vascular developments at P14.

Conclusions Early postnatal systemic LPS induced systemic and pulmonary pro-inflammatory responses and disrupted alveolar and pulmonary vascular developments. This rat model of new BPD induced by early postnatal inflammation per se without an exposure to hyperoxia can be used to test the effects of antiinflammatory agents for BPD.

\section{PS-211a TOTAL OXIDANT STATUS AND TOTAL ANTIOXIDANT CAPACITY IN AMNIOTIC FLUID OF LATE PRETERM INFANTS}

${ }^{1} \mathrm{I}$ Mungan Akin, ${ }^{1} \mathrm{MS}$ Yanartas, ${ }^{1} \mathrm{~S}$ Sevuk Ozumut, ${ }^{2} \mathrm{~B}$ Isbilen, ${ }^{1} \mathrm{D}$ Buyukkayhan, ${ }^{2} \mathrm{~F}$ Isman. ${ }^{1}$ Department of Pediatrics, Istanbul Medeniyet University Goztepe Education and Research Hospital, Istanbul, Turkey; ${ }^{2}$ Department of Biochemistry, Istanbul Medeniyet University Goztepe Education and Research Hospital, Istanbul, Turkey

\subsection{6/archdischild-2014-307384.510}

Background and aims Pregnancy represents a complex state in which the mother and the fetus both contribute to the oxidative stress and production of reactive oxygen species. The ability to buffer these is called total antioxidant capacity (TAC). We aimed to evaluate whether amniotic total oxidant status (TOS) and TAC can have a role in late preterm births after premature rupture of membranes (ROM).

Methods The study was a prospective observational trial. Group 1 ( $\mathrm{n}=30)$ : late preterms with premature ROM, Group 2 ( $\mathrm{n}=$ 30): term infants with prolonged ROM, Group 3 ( $\mathrm{n}=30$ ): term infants born without prolonged ROM. Amniotic fluid and cord blood samples were collected during delivery and TOS $\left(\mu \mathrm{molH}_{2} \mathrm{O}_{2} \mathrm{Eq} / \mathrm{L}\right)$ and $\mathrm{TAC}(\mathrm{mmol}$ Trolox Eq/L) levels were measured.

Results Mean gestational ages were 35,3 \pm 1,1; 39,29 \pm 1,25; $38,7 \pm 0,63$ weeks respectively. Cord blood samples of the groups revealed no difference between any of the parameters checked $\left(\mathrm{pH}, \mathrm{pCO}_{2}, \mathrm{HCO}_{3}\right.$, lactate, Methb, TAC and TOS) ( $\mathrm{p}>$ 0.05 , for each). TAC levels of amniotic fluids $(2,05 \pm 0,60$; $2,04 \pm 0,5$ and $21,89 \pm 0,36)$ were also similar between groups $(\mathrm{p}>0.05)$. But TOS levels of Group1 was significantly higher than Group2 $(\mathrm{p}<0.05)$ and Group3 ( $<<0.01)$. No significant difference was detected between Group 2 and 3.
Conclusion Similar cord blood levels of all parameters reflect the similar perinatal conditions of infants at birth. Similar TOS levels of term groups show that there is no close relation between TOS and infectious status. But on the other hand; significantly higher TOS levels of Group1 can be related with premature birth; which can lead us to antioxidant therapies to avoid premature birth.

\section{Neonatal Sepsis}

\section{PS-212 EPIDEMIOLOGY OF NEONATAL SEPSIS IN SWITZERLAND - RESULTS FROM THE SWISS PAEDIATRIC SEPSIS STUDY}

${ }^{1} \mathrm{E}$ Giannoni, ${ }^{2} \mathrm{LJ}$ Schlapbach, ${ }^{2} \mathrm{P}$ Agyeman, ${ }^{3} \mathrm{~K}$ Posfay-Barbe, ${ }^{4} \mathrm{~A}$ Donas, ${ }^{5} \mathrm{U}$ Heininger, ${ }^{6} \mathrm{G}$ Konetzny, ${ }^{7} \mathrm{P}$ Hasters, ${ }^{8} \mathrm{~A}$ Niederer-Loher, ${ }^{9} \mathrm{~W}$ Baer, ${ }^{10} \mathrm{C}$ Relly, ${ }^{2} \mathrm{~B}$ Wagner, ${ }^{10} \mathrm{C}$ Aebi, ${ }^{10} \mathrm{C}$ Berger. 'Service of Neonatology, Centre Hospitalier Universitaire Vaudois and University, Lausanne, Switzerland; '2Department of Pediatrics, University Children's Hospital Bern, Bern, Switzerland; ' ${ }^{3}$ Département de l'Enfant Et de l'Adolescent, Hôpitaux Universitaires de Genève, Geneva, Switzerland; ${ }^{4}$ Department of Pediatrics, Kinderspital Luzern, Luzern, Switzerland; ${ }^{5}$ Infektiologie Und Vakzinologie, Universitäts-Kinderspital Beider Basel, Basel, Switzerland; ${ }^{6}$ Kinderklinik, Kantonsspital Aarau, Aarau, Switzerland; ${ }^{7}$ Klinik Für Neonatologie, UniversitätsSpital Zürich, Zurich, Switzerland; ${ }^{8}$ Ostschweizer Kinderspital, Infektiologie, St. Gallen, Switzerland; ${ }^{9}$ Kantonsspital Graubünden, Kinderklinik Chur, Chur, Switzerland; ${ }^{10}$ Division of Infectious Diesases, University Children's Hospital Zürich, Zurich, Switzerland

\subsection{6/archdischild-2014-307384.511}

Background and aims Neonatal infection is a major cause of morbidity and mortality. The ongoing Swiss Paediatric Sepsis Study prospectively evaluates the epidemiology of blood cultureproven sepsis in newborns and children in Switzerland.

Methods Newborn infants admitted to nine Swiss neonatal intensive care units (NICUs) and presenting with culture-proven sepsis between 9.2011-2.2014 were prospectively enrolled. Early-onset sepsis (EOS) was defined as infection occurring.

Results We identified 189 episodes of blood culture-proven sepsis in 186 patients. Thirty seven episodes were classified as EOS and 152 episodes were classified as LOS. Median gestational age at birth was 34 weeks for EOS, and 28 weeks for LOS. Mortality was $14 \%$ in EOS and $9 \%$ in LOS. Forty six percent of patients required mechanical ventilation during the sepsis episode, and $4 \%$ required catecholamine treatment for arterial hypotension.

Group B Streptococcus (GBS) and Escherichia coli were the most frequently isolated pathogens in EOS, accounting for $35 \%$ and $16 \%$ of episodes. Coagulase-negative staphylococci were the leading pathogens in LOS (36\%), followed by Staphylococcus aureus (18\%), Escherichia coli (16\%), and GBS (11\%). The proportion of hospital-acquired LOS due to Coagulase-negative staphylococci, Staphylococcus aureus and Escherichia coli varied between $10-68 \%, 0-45 \%$ and $0-30 \%$ in different NICUs.

Conclusions This national study confirms that neonatal sepsis continues to cause high morbidity and significant mortality. GBS is the most common cause of EOS. There are important differences in the aetiology of LOS in different institutions.

\section{PS-213 EPIDEMIOLOGY AND OUTCOME OF NEONATAL SEPTIC SHOCK IN A PICU OF TUNISIA}

A Borgi, M Chamem, S Mahdoui, N Ghali, S Belhadj, A Khaldi, A Hamdi, K Menif, A Bouziri, N Benjaballah. PICU, Children's Hospital Béchir Hamza, Tunis, Tunisia

10.1136/archdischild-2014-307384.512 
Background Neonatal septic shock is a devastating condition associated with high morbidity and mortality.

Methods A retrospective study was conducted in children's hospital Tunisian PICU between 2005 to 2013. All neonates $(<28$ days) treated for septic shock with bacterial proof were included. Nosocomial infection was an exclusion criteria. The chart review relieved demographics, length of stay, therapies and outcomes.

Results A total of 40 neonates were included. Mean age on admission was $34 \mathrm{~h} \pm 3.7$. Mean SNAPP score was $25 \pm 17$. Materno-fetal infection was observed in 37 cases, staphylococcal pneumonia in 2 cases and bacterial coinfection with bronchiolitis in the last case. The bacteriological study showed a notable predominance of streptococcus B (40\%) and E.coli (35\%). All patients required mechanical ventilation (mean duration: $85 \pm$ $556 \mathrm{~h}$ ) and haemodynamic support (mean duration $49 \pm 335$ h). Mortality rate was $19 \%$ in full term infants, $12.5 \%$ in near term infants and $27 \%$ in extremely preterm infants.

Conclusions Our results would indicate a high mortality rate in neonatal septic shock. A goal -directed therapy for septic shock, implanted in our PICU, could improve outcomes for this vulnerable population.

\section{PS-214 DOES A TOTAL STERILE COLLECTION BUNDLE REDUCE FALSE POSITIVE BLOOD CULTURE RATES AND ANTIBIOTIC USE IN NEONATAL INTENSIVE CARE?}

HE Gillett, LF Hamilton, JW Davis. Neonatal Intensive Care, St Michaels Hospital, Bristol, UK

10.1136/archdischild-2014-307384.513

Background and aim In neonatal intensive care coagulase negative Staphylococcus species can be both blood culture contaminant and pathogen. False positive cultures can result in clinical uncertainty and unnecessary antibiotic use. Our aim was to assess the effect of a total sterile blood culture collection bundle on the incidence of false positive blood cultures in a regional surgical neonatal intensive care unit.

Method Clinical data of all infants who had blood cultures taken before and after the introduction of the collection bundle (sterile technique and 2\% Chlorhexidine) were collected. The rates of false positive blood cultures, defined as the presence of a skin commensal and $<3$ predefined clinical signs (Modi et al. 2009), were compared.

Results In total 367 blood cultures from 294 babies were assessed, 197 pre-intervention (PRE) and 170 following bundle introduction (POST). The median birth weight and gestation were similar in both groups. The rate of false positive cultures in the total PRE group was $9 / 197(4.6 \%)$ compared to $1 / 170$ $(0.6 \%)$ in the POST group ( $<<0.05)$. In infants $<28$ weeks' the rates reduced from $4 / 29(13.8 \%)$ to $0 / 30(0 \%)$ ( $p<0.05)$. Unnecessary antibiotic exposure rate was $7.7 \%$ in the PRE group versus $0.0 \%$ in the POST group $(\mathrm{p}<0.05)$.

Conclusion Implementation of this collection bundle reduced the number of false positive blood culture results. This has a potential benefit in reducing unnecessary antibiotic use and associated health care costs.

\section{PS-215 SEPSIS REDUCTION CARE BUNDLES IMPROVE COGNITIVE OUTCOME IN VERY LOW BIRTH WEIGHT INFANTS}

${ }^{1} \mathrm{JW}$ Davis, ${ }^{2} \mathrm{~S} J a r y,{ }^{1} \mathrm{PA}$ Cairns, ${ }^{1} \mathrm{D}$ Harding, ${ }^{2} \mathrm{~K}$ Luyt. ${ }^{1}$ Neonatology, St. Michael's Hospital University Hopsitals Bristol, Bristol, UK; ${ }^{2}$ Neonatal Neuroscience, University of Bristol, Bristol, UK
Background Very low birth weight (VLBW) infants with late onset sepsis have increased risk of neurodisability. Care bundles to reduce these infections in NICU are effective. The impact of care bundles on long-term neurodevelopmental outcome has not been described. We aimed to determine if implementation of a sepsis-reduction care bundle was associated with improvement in neurodevelopmental outcomes in VLBW infants.

Methods A multimodal sepsis improvement bundle was implemented in a regional NICU from July 2006. This bundle focused on hand hygiene and line care improvements. Mortality and neurological morbidity rates were compared pre- and post intervention (Jan '01 - Dec '07 vs. Jul '08 - Dec '12). Infants had neurodevelopmental assessment at 24 months corrected gestation with Bayley Scales of Infant development. Moderate cognitive disability was defined as a cognitive/language score below 2SDs, moderate motor disability as a motor score below 2SDs.

Results Birth weight, gestation and gender were similar in both cohorts. Coagulase Negative Staphylococcus septicaemia rates reduced from $7 / 1000$ care days before implementation to 2.8 / 1000 in 2012. Mortality rates were similar between the groups (66/426 vs. $40 / 310 ; p=0.3)$. There was no difference in moderate motor disability ( $17 / 85$ vs. $3 / 42 ; \mathrm{p}=0.07)$. There was a significant reduction in moderate cognitive disability (16/86 vs. $2 / 44 ; p=0.03$ ) after implementation of the sepsis care bundle.

Conclusions Sepsis-reduction care bundles improve the 2-year neurodevelopmental outcome of VLBW infants. The improvement seen in cognitive function is likely to translate into significantly less long-term learning disability.

\section{PS-216 MULTICENTRE PROSPECTIVE STUDY IN THE MANAGEMENT OF PRESUMED OR CONFIRMED EARLY ONSET SEPSIS AND INITIATION OF TREATMENT IN THE TERM AND PRETERM NEONATAL ADMISSIONS}

${ }^{1} \mathrm{M}$ Chalia, ${ }^{2} \mathrm{M}$ Upton. ${ }^{1}$ Neonatal UnitCambridge University Hospitals NHS Foundation Trust Addenbrookes Hospital, Cambridge, UK; ${ }^{2}$ Neonatal Network, East of England Deanery, Cambridge, UK

\subsection{6/archdischild-2014-307384.515}

Aim To assess the choice of antibiotics in correlation to clinical management of suspected or confirmed early onset neonatal sepsis. To identify the requirement for updating our practice within the East of England Neonatal Network.

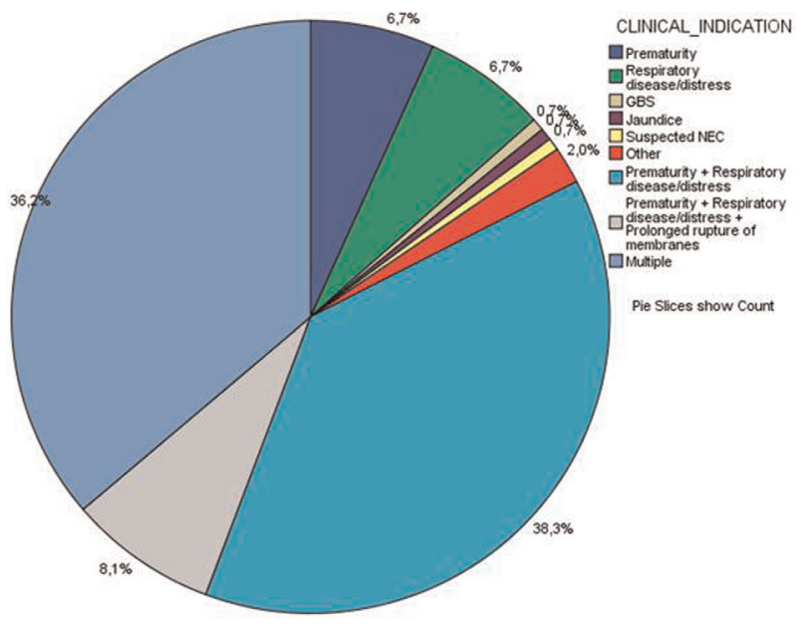

Abstract PS-216 Figure 1 